\title{
ISOMETRIC IMMERSIONS WHICH PRESERVE CURVATURE OPERATORS
}

\author{
BARRETT O'NEILL
}

The curvature tensor of a Riemannian manifold $M$ can be expressed by a function which assigns to each pair of vectors $x, y \in M_{m}$ (tangent space to $M$ at $m$ ) a skew-symmetric linear operator $R_{x y}$ on $M_{m}$ [1]. Call $R_{x y}$ the curvature operator of $x, y$. Let $j: M^{d} \rightarrow \bar{M}^{d+1}$ be an isometric immersion. If $j$ is totally geodesic, then $j$ preserves curvature operators, that is, if $x, y, z \in M_{m}$, then $\operatorname{dj}\left(R_{x y}(z)\right)=\bar{R}_{d j(x), d j(y)}(d j(z))$. The converse is generally false. We are going to consider the character of immersions as above which preserve curvature operators. The simplest example is an arbitrary isometric immersion of $R^{d}$ in $R^{d+1}$. In particular we show that if the domain $M^{d}$ of $j$ is complete and has positive curvature then the converse above holds, that is, if $j$ preserves curvature operators, then $j$ is totally geodesic.

1. General case. Note that $j: M^{d} \rightarrow \bar{M}^{d+1}$ preserves curvature operators if and only if (a) $j$ preserves Riemannian curvature, i.e. $\bar{K}(d j(\pi))=K(\pi)$ for all 2-planes $\pi$ tangent to $M$, and (b) if $z \in \bar{M}_{j(m)}$ is orthogonal to $d j\left(M_{m}\right)$, then $\bar{R}_{d j(x), d j(y)}(z)=0$ for all $x, y \in M_{m}$. The proof is elementary, and depends on the fact that the codimension of $M$ in $\bar{M}$ is one.

THEOREM 1. Let $j: M^{d} \rightarrow \bar{M}^{d+1}$ be an isometric immersion which preserves curvature operators, and let $M$ be complete. Then the open set $N$ of nongeodesic points of $M$ rel. $j$ is foliated by complete $(d-1)$-dimensional submanifolds which are totally geodesic rel. $j$.

Proof. Since $j$ preserves Riemannian curvature, at each point of $M$ there is at most one curvature direction with nonzero principal curvature. Thus on the set $N$ of nongeodesic points, the directions of zero normal curvature constitute a differentiable field $\beta$ of $(d-1)$ planes. We will integrate $\rho$ to obtain the required foliation. (The theorem holds trivially when $N$ is empty.)

Each point of $N$ has a neighborhood $U$ on which there is a unit normal vector field $E_{d+1}$ rel. $j$ and a frame field $E=\left(E_{1}, \cdots, E_{d}\right)$ whose first vector is in the curvature direction with principal curvature $\kappa_{1} \neq 0$. From the frame field $E$ one obtains on $U$ the dual-base forms $\omega_{i}$, the Riemannian connection forms $\phi_{i j}$, and curvature forms $\Phi_{i j}$ of $M, 1 \leqq i, j \leqq d$. Enlarging $E$ by adding $E_{d+1}$ to it, we get the Codazzi forms $\sigma_{i}, 1 \leqq i \leqq d$, and curvature forms $\bar{\Phi}_{r s}, 1 \leqq r, s \leqq d+1$,

Received by the editors July $11,1961$. 
of $\bar{M}$. Dropping the differential map of $j$ from the notation, we can write $\bar{R}_{E_{i} E_{j}}\left(E_{d+1}\right)=-\sum_{k} \bar{\Phi}_{k, d+1}\left(E_{i}, E_{j}\right) E_{k}$. Thus by (b) above, we have $\bar{\Phi}_{k, d+1}=0$ on $U$. Furthermore, $\sigma_{1}=\kappa_{1} \omega_{1} \neq 0$, and $\sigma_{i}=0$ if $i>1$. Thus the Codazzi equations $d \sigma_{i}=-\sum_{k} \phi_{i k} \wedge \sigma_{k}+\bar{\Phi}_{d+1, i}$ reduce to $d \sigma_{1}=0$ and $\phi_{i 1} \wedge \sigma_{1}=0$. Since $\sigma_{1}$ annihilates the planes of $\rho, d \sigma_{1}=0$ implies $P$ is integrable. The other equations imply that the forms $\phi_{i 1}$ are zero on vectors tangent to a leaf $L$ of $\beta$. But these forms, $1<i \leqq d$, are the Codazzi forms for $L$ in $M$, so each leaf $L$ is totally geodesic in $M$-and hence also in $\bar{M}$, i.e. rel. $j$.

Now we show that the leaves $L$ are complete by showing that geodesics of $L$ are infinitely extendible. Suppose the contrary, i.e. that there is a maximal geodesic $\alpha$ of a leaf $L$ which is defined only on a bounded open interval $(a, b)$. Since $M$ is complete, $\alpha$ is infinitely extendible as a geodesic of $M$. Since $L$ is totally geodesic; as long as this extension $\tilde{\alpha}$ remains in $N$, it is a geodesic of $L$. So the limit points $\tilde{\alpha}(a)$ and $\tilde{\alpha}(b)$ of $\alpha$ are not in $N$. We will contradict this by showing that $\kappa_{1}$ is zero at neither of these points. We can assume that the geodesic segment $\alpha$ (but not its limit points) lies in the domain of fields $E$ and $E_{d+1}$ as above, with the further properties that $\alpha$ is an integral curve of $E_{2}$ and that $E$ is parallel on $\alpha$. In fact, once $E$ is properly defined on $\alpha$, one can extend over a neighborhood of $\alpha$ in $M$ by first extending over a neighborhood in the leaf $L$, keeping $E_{1}$ perpendicular to $L$, then extending over the full neighborhood, keeping $E_{1}$ always in the $\kappa_{1}$ curvature direction. (Strictly speaking, one passes to a suitable covering manifold if $\alpha$ crosses itself.)

From the first structural equation, we deduce $\left[E_{1}, E_{2}\right]=\sum \phi_{i 2}\left(E_{1}\right) E_{i}$. Applying the form $d \sigma_{1}=0$ to the fields $E_{1}, E_{2}$ gives $E_{2}\left(\kappa_{1}\right)=-\kappa_{1} \phi_{12}\left(E_{1}\right)$. Setting $k=\kappa_{1} \circ \alpha, f=\phi_{12}\left(E_{1}\right) \circ \alpha$, we write this equation as

$$
k^{\prime}=-k f \text {. }
$$

Applying the second structural equation to the fields $E_{1}, E_{2}$ and simplifying, using the facts above, we get $E_{2}\left(\phi_{12}\left(E_{1}\right)\right)=-\left(\phi_{12}\left(E_{1}\right)\right)^{2}$ $-\Phi_{12}\left(E_{1}, E_{2}\right)$. Setting $F=\Phi_{12}\left(E_{1}, E_{2}\right) \circ \alpha$ yields

$$
f^{\prime}=-f^{2}-F \text {. }
$$

Our assumption that $L$ is not complete has led to the conclusion that $k(t)$ approaches zero as $t$ approaches either $a$ or $b$. The differential equations (1) and (2) contradict this. In fact, solving (1) explicitly, we deduce that as $t \rightarrow b, \lim \sup f=+\infty$. This contradicts (2) which says, since $F$ is bounded below on $(a, b)$, that when $f$ is large enough its slope is negative. The argument when $t \rightarrow a$ is similar, so the proof is complete. 
A scheme similar to that above was used by Chern and Lashot in [3, Lemma 2].

TheOREM 2. Suppose $M^{d}(d \geqq 2)$ is complete and has Riemannian curvature $K>0$. Then every isometric immersion $j: M^{d} \rightarrow \bar{M}^{d+1}$ which preserves curvature operators is totally geodesic.

Proof: Suppose there is a nongeodesic point, that is (in the notation of the previous proof) $N$ is not empty. Then a geodesic $\alpha$ as in that proof has domain the whole real line. Thus we can arrange for the function $f=\phi_{12}\left(E_{1}\right) \circ \alpha$ to be defined on the whole real line, and $f$ satisfies the differential equation (2) $f^{\prime}=-f^{2}-F$. But this is impossible when $K>0$, since then $F>0$.

This is not a local result-it fails if $M$ is not required to be complete.

2. Constant curvature case. If $\bar{M}^{d+1}$ has constant curvature, then its curvature operators have the property that $\bar{R}_{x y}(z)=0$ if $z$ is perpendicular to $x$ and $y$. (Converse, $\$ 177$ of [2].) Thus by the first remark of the previous section, if $M^{d}$ and $\bar{M}^{d+1}$ have the same constant curvature, then every isometric immersion $j: M^{d} \rightarrow \bar{M}^{d+1}$ preserves curvature operators. We consider the character of $j$ and $M^{d}$ when $\bar{M}^{d+1}$ is specialized to be a sphere $S^{d+1}(C)$, Euclidean space $R^{d+1}$, or hyperbolic space $Q^{d+1}(C)$, where $C$ is curvature of appropriate sign. From Theorem 2 we get: if $M^{d}$ is complete and has constant curvature $C>0$, then $M^{d}$ can be immersed in $S^{d+1}(C)$ if and only if $M^{d}$ is isometric to $S^{d}(C)$. Any such immersion is an imbedding onto a great $d$-sphere.

In the case $C=0$, Hartman and Nirenberg [4] have proved: $a$ complete flat manifold $M^{d}$ can be immersed in $R^{d+1}$ if and only if $M^{d}$ is isometric to either $R^{d}$ or $S^{1}(r) \times R^{d-1}$. Any such immersion is as a cylinder in $R^{d+1}$.

This can be proved by applying Theorem 1 to both $j: M^{d} \rightarrow R^{d+1}$ and $j \circ \pi: R^{d} \rightarrow R^{d+1}$, where $\pi: R^{d} \rightarrow M^{d}$ is the universal covering of $M^{d}$. The special character of disjoint, totally geodesic hypersurfaces in $R^{d}$ allows us to extend the foliation of the set $N$ in $R^{d}$ to a foliation of all of $R^{d}$ by parallel $(d-1)$-planes.

This general scheme fails in the negative curvature case, since disjoint, totally geodesic hypersurfaces in $Q^{d}(C)$ can have more complicated arrangements. One can exhibit surfaces with curvature $C<0$ in $Q^{3}(C)$ with arbitrary first Betti number. However the Euclidean result can be extended topologically to the negative curvature case as follows: 
TheOREM 3. Let $M^{d}$ be a complete manifold with constant negative curvature $C$. If $M^{d}$ can be isometrically immersed in $Q^{d+1}(C)$, then $H^{i}\left(M^{d}\right)=0$ for $i \geqq 2$.

(Here $H$ denotes Cech cohomology with arbitrary coefficients.)

Proof. From such an immersion $j$ we get a decomposition of $M$ as in Theorem 1. Denote the components of $N$ by $N_{\alpha}$, the components of $M-N$ by $F_{\beta}$. Each leaf $L$ of $N$ is complete and totally geodesic rel. $j$, hence isometric to $Q^{d-1}=Q^{d-1}(C)$. The immersion $j$ is one-toone on components $F_{\beta}$ also. Let $\pi: Q^{d} \rightarrow M^{d}$ be the universal covering. Then we can derive

(1) If a subset $A$ of $M$ can be lifted into $Q^{d}$, so can the union of those sets $L$ and $F_{\beta}$ which meet $A$.

(2) There is a number $\epsilon>0$ such that if $B, C, D$ are disjoint totally geodesic hypersurfaces in $Q^{d}$ which meet an $\epsilon$-neighborhood, then $B, C, D$ are linearly ordered, i.e. some one separates the other two in $Q^{d}$.

(3) Each $F_{\beta}$ is either a totally geodesic $Q^{d-1}$ or (if its interior is not empty) a manifold with boundary $B_{\beta}$, where $B_{\beta}$ is a union of totally geodesic sets $Q^{d-1}$, each of which is disjoint from the closure of the others. In particular each $F_{\beta}$ is contractible.

By a theorem of Ricci ( $\$ 107,[2])$ the orthogonal trajectories of the leaves of an $N_{\alpha}$ give isometries of the leaves. If $N$ is dense in $M$ it follows (much as in the Euclidean case) that $M$ is diffeomorphic to either $R^{d}$ or $S^{1} \times R^{d-1}$. Excluding this case we have

(4) The boundary of each $N_{\alpha}$ is either a single totally geodesic $Q^{d-1}$ or two disjoint ones, and the closure $\bar{N}_{\alpha}$ of $N_{\alpha}$ is contractible.

Consider the covering $\mathfrak{e}$ of $M$ by all sets $N_{\alpha}$ and $F_{\beta}$. This is a closed covering by homologically trivial sets. Furthermore, any intersection of three elements of $\mathfrak{C}$ is empty, and the intersection of any two consists of at most two disjoint sets $Q^{d-1}$. Suppose $\mathcal{C}$ is locally finite, e.g. $M-N$ only a finite number of components. Then by a well-known theorem, the cohomology of $M$ is isomorphic to the cohomology of the nerve of $\mathfrak{e}$. Since this nerve has dimension 1 the result follows. If $\mathfrak{e}$ is not locally finite we can alter it, retaining its essential properties, so as to get local finiteness. We omit the details of the proof. Roughly speaking, if $\mathfrak{e}$ is not locally finite at a point $p$, then $p$ lies in a "limit face" $Q_{1}$ of an element, say $\bar{N}_{\alpha}$, of $\mathcal{e}$. Choose $N_{\beta} \neq N_{\alpha}$ sufficiently near $Q_{1}$ and let $Q_{2}$ be the face of $N_{\beta}$ nearest $Q_{1}$. Using (1) and (2) we can define $G$ to be the union of $Q_{1}, Q_{2}$, and the elements of $\mathcal{C}$ between $Q_{1}$ and $Q_{2}$. Finally, replace these elements by $G$ in $\mathcal{C}$. Iteration of this operation eliminates all limit faces.

In general the complexity of the decomposition of $M$ given by 
Theorem 1 is measured by the identification space $M^{*}$ whose elements are the leaves of $N$ and the components of $M-N$. If $M^{*}$ is metrizable, it can be shown to have inductive dimension 1 . In this case the argument above can be replaced by an application of the Vietoris mapping theorem.

\section{BIBLIOGRAPHY}

1. W. Ambrose and I. M. Singer, A theorem on holonomy, Trans. Amer. Math. Soc. 75 (1953), 428-443.

2. E. Cartan, Leçons sur la géométrie des espaces de Riemann, Gauthier-Villars, Paris, 1951.

3. S. Chern and R. Lashof, On the total curvature of immersed manifolds, Amer. J. Math. 79 (1957), 306-318.

4. P. Hartman and L. Nirenberg, On spherical image maps whose Jacobians do not change sign, Amer. J. Math. 81 (1959), 901-920.

University of California, Los Angeles

\section{ON THE EMBEDDABILITY OF THE REAL PROJECTIVE SPACES ${ }^{1}$}

\section{MARK MAHOWALD ${ }^{2}$}

In a paper of the same title, Massey [4] proved that if $2^{k-1}+2^{k-9}$ $-1 \leqq n<2^{k}$ then $P_{n}$ cannot be differentiably embedded in $R^{2^{k}}$. By using the technique of Massey in a different way we can prove the following theorem which clearly includes Massey's.

TheOREM. If $2^{k-1}<n<2^{k}$ then $P_{n}$ cannot be embedded differentiably in Euclidean space of dimension $2^{k}$.

Besides the result of Massey, the main result in this direction is if $2^{k-1}<n<2^{k}$ then $P_{n}$ cannot be embedded differentiably in $R^{2^{k}-1}$. Our result yields, in particular, that for $P_{2^{k}+1}$, the embedding in $R^{2^{k+1}+1}$ given by Hopf and James [1] is the best possible.

The following information from $[3 ; 4]$ will be needed. Let $M$ be a $n$-manifold differentiably embedded in $R^{n+k+1}$; and let $p: E \rightarrow M$ denote the bundle of unit normal vectors. Then there exist subalgebras $A^{*}(E, Z) \subset H^{*}(E, Z)$ and $A^{*}\left(E, Z_{2}\right) \subset H^{*}\left(E, Z_{2}\right)$ which satisfy the following conditions:

1. $A^{0}(E, G)=H^{0}(E, G)$,

2. $H^{q}(E, G)=A^{q}(E, G)+p^{*}\left(H^{q}(B, G)\right)(0<q<n+k)$,

3. $A^{q}(E, G)=0, q \geqq n+k$,

Received by the editors October 2, 1961.

${ }^{1}$ Sponsored by the U. S. Army Research Office (Durham).

2 The referee has informed me that the result of this paper has been obtained independently by Mr. J. P. Levine in his thesis at Princeton University. 